POS PROCEEDINGS

\title{
A light singlino in the NMSSM: Challenges for SUSY searches at the LHC
}

\section{Ulrich Ellwanger*}

LPT, University Paris-Sud and School of Physics and Astronomy, University of Southampton

E-mail: ellwangerath.u-psud. fr

\begin{abstract}
A light singlino in the NMSSM can reduce considerably the missing transverse energy at the end of sparticle decay cascades; instead, SM-like or light NMSSM-specific Higgs bosons can be produced. Such scenarios can be consistent with present constraints from the LHC with all sparticle masses below $\sim 1 \mathrm{TeV}$. We discuss search strategies, which do not rely on missing transverse energy, for such scenarios at the next run of the LHC near $14 \mathrm{TeV}$.
\end{abstract}

The European Physical Society Conference on High Energy Physics

22-29 July 2015

Vienna, Austria

${ }^{*}$ Speaker. 


\section{Introduction}

Supersymmetric extensions of the Standard Model (SM) can solve simultaneaously several shortcomings of the SM: The hierarchy problem can be strongly reduced, the presence of dark matter can be explained, and the running gauge couplings are automatically consistent with a Grand Unified Theory (GUT).

Hence, despite the missing discovery of supersymmetric particles (sparticles) at the run I of the LHC, it is hard to give up this attractive extension of the SM - the more so given that no hints for other physics beyond the SM, solving the above problems, have been observed.

In the framework of the Minimal Supersymmetric extension of the SM (MSSM) the absence of sparticles (squarks, gluinos) with masses below $\sim 1 \mathrm{TeV}$ generates a "little hierarchy problem" meaning that some finetuning among its parameters is required in order to explain a weak scale about a factor 10 below the sparticle masses. In fact, assuming $M_{\text {squark }} \sim M_{\text {gluino }}$ (for the squarks of the first generations), a LSP mass below $500 \mathrm{GeV}$ and simple decay cascades, the lower limits on squark/gluino masses are about $\sim 1.7 \mathrm{TeV}$ as indicated in Fig. 1 from [1]. (Similar bounds have been obtained by CMS.)

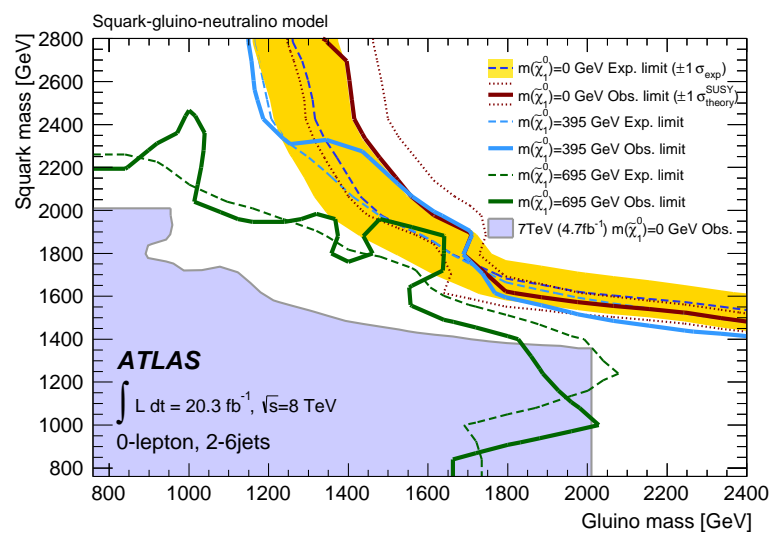

Figure 1: Lower bounds on squark/gluino masses obtained by ATLAS (from [1]). $\chi_{1}^{0}$ denotes the LSP.

However, these lower limits rely on one of the standard signatures of sparticle decay cascades: missing transverse energy $\left(E_{T}^{\text {miss }}\right)$ carried away by the lightest supersymmetric particle (LSP) if Rparity is conserved. In the MSSM the last step NLSP $\rightarrow \mathrm{LSP}+\mathrm{X}$ in a sparticle decay cascade gives always a sizeable energy to the LSP which subsequently escapes its detection.

The Next-to-Minimal Supersymmetric SM (NMSSM) [2] shares the benefits of supersymmetric extensions of the Standard Model (SM) with the MSSM. In addition the $\mathbb{Z}_{3}$-invariant version of the NMSSM (with a scale invariant superpotential) solves the $\mu$-problem of the MSSM. Both the general and the $\mathbb{Z}_{3}$-invariant versions of the NMSSM render more natural the mass of $\sim 125 \mathrm{GeV}$ of the SM-like Higgs boson $H_{S M}$. For these reasons the NMSSM has become more and more appealing in the recent years.

The field content of the NMSSM differs from the MSSM by an additional gauge singlet superfield $\tilde{S}$ which contains a Majorana fermion (the singlino), a CP-even and a CP-odd scalar. The 
couplings of the components of $\tilde{S}$ to the MSSM-like Higgs fields $H_{u}, H_{d}$ and sparticles are proportional to a dimensionless coupling $\lambda$, and the self couplings of the components of $\tilde{S}$ are proportional to a dimensionless coupling $\kappa$.

The singlino of the NMSSM can well be the LSP and a good dark matter candidate with a relic density consistent with present bounds. This remains true of $\lambda$ is relatively small $\left(\sim \mathscr{O}\left(10^{-2}\right)\right)$ in which case the singlino couples only weakly to all other sparticles. Consequently no sparticle wants to decay into the singlino - except for the NLSP which has no other choice (due to R-parity conservation), see Fig 2.

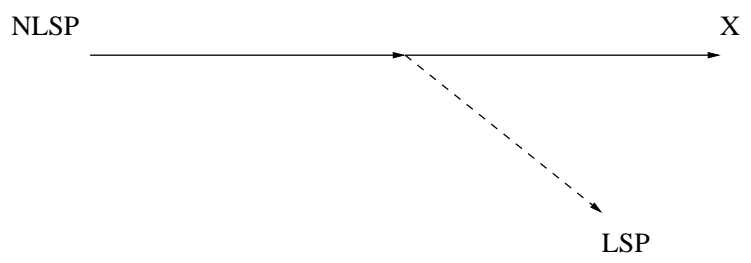

Figure 2: Scetch of a last step NLSP $\rightarrow$ LSP + X of a sparticle decay cascade

Let us consider the (natural) configuration where the singlino LSP is light (a few $\mathrm{GeV}$ ), $X \equiv H$ is a Higgs boson $\left(H_{S M}\right.$, or an additional NMSSM-specific scalar $\left.H_{S}\right)$, and the mass of the NLSP is not far above $M_{\text {singlino }}+M_{H}$. Then it follows for kinematical reasons that little (missing transverse) energy is transferred from the NLSP to the singlino; the transverse energy is carried away by the Higgs boson. Hence, if Higgs decays do not give rise to $E_{T}^{\text {miss }}$, the $E_{T}^{\text {miss }}$ signature is dramatically reduced! (Note that NMSSM-specific Higgses $H_{S}$ with masses below $125 \mathrm{GeV}$ are not ruled out by LEP if their couplings to Z-bosons are sufficiently small; such light $H_{S}$ can also play the rôle of " $X$ " in NLSP $\rightarrow X+$ singlino cascades.)

In [4] we have studied the impact of such a scenario for squark/gluino searches at the run I of the LHC at $8 \mathrm{TeV}$ c.m. energy. We compared, amongst others, the spectrum of $E_{T}^{\text {miss }}$ of the production of squarks/gluinos of masses of $\sim 1 \mathrm{TeV}$

a) in the MSSM with a $\sim 89 \mathrm{GeV}$ bino-like LSP, to

b) the NMSSM where the bino decays into a $\sim 5 \mathrm{GeV}$ singlino and a NMSSM-specific Higgs scalar $H_{S}$ of $\sim 83 \mathrm{GeV}$. (It was checked that these masses, branching fractions and a good relic density can be obtained for suitable parameters.)

The resulting spectra are shown in the Fig 3 for the MSSM in red, and for the NMSSM in blue. The tremendous decrease of $E_{T}^{\text {miss }}$ is obvious. (The remaining $E_{T}^{\text {miss }}$ for the NMSSM originates essentially from neutrinos from $H_{S}$ decays into $\tau \tau$ or $b b$.)

The corresponding MSSM-scenario would have been ruled out by ATLAS and CMS by standard searches for jets and $E_{T}^{\text {miss }}$. Applying the corresponding cuts and upper limits on signal rates we found that a NMSSM-scenario with squark masses of $\sim 830 \mathrm{GeV}$, gluino masses of $\sim 860 \mathrm{GeV}$ (and stop and chargino masses just below $1 \mathrm{TeV}$ in order to prevent neutrinos from squark/gluino decay cascades) passes the present constraints - this is the only scenario allowed after the run I of the LHC with all sparticle masses below $1 \mathrm{TeV}$.

Let us comment on why the kinematical configuration $M_{\mathrm{NLSP}} \sim M_{\mathrm{Higgg}}, M_{\mathrm{LSP}} \ll M_{\mathrm{NLSP}}$ cannot reduce significantly the spectrum of $E_{T}^{\text {miss }}$ in the MSSM: The LSP would have to be bino-like, and 


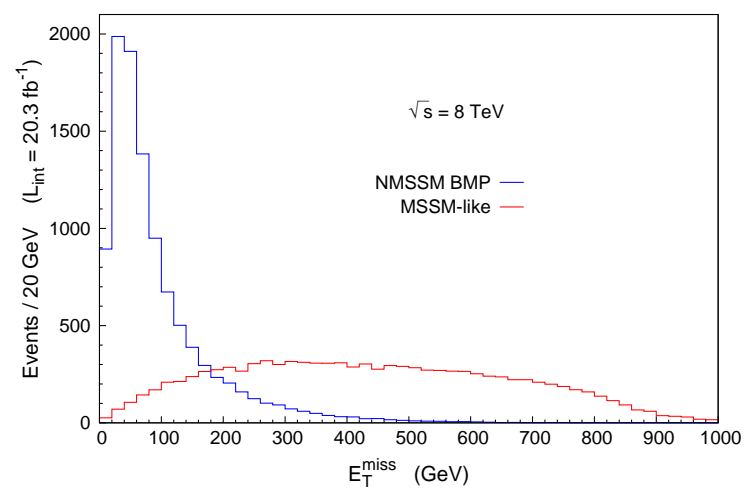

Figure 3: Spectra of $E_{T}^{\text {miss }}$ before cuts for a scenario in the MSSM with a $\sim 89 \mathrm{GeV}$ bino-like LSP (red), and in the NMSSM where the bino decays into a $\sim 5 \mathrm{GeV}$ singlino and a NMSSM-specific Higgs scalar $H_{S}$ of $\sim 83 \mathrm{GeV}$.

the bino has sizeable couplings to squarks/quarks. Hence squarks decay with a sizeable branching fraction directly into quarks + bino, without an NLSP in the squark decay cascade. These events will thus lead to large $E_{T}^{\text {miss }}$ as usual and - even if not all squarks decay this way - allow for sparticle detection via the standard cuts on $E_{T}^{\text {miss }}$.

Thus we have to study how sparticles (notably squarks/gluinos with the largest production cross sections) can be observed at the run II of the LHC without relying on the signature of large $E_{T}^{\text {miss }}$. Since $E_{T}^{\text {miss }}$ is replaced by the final state $M_{\text {singlino }}+M_{H}$ in each sparticle decay cascade, the presence of two Higgs bosons in each event of squark/gluino pair production (on top of the usual hard jets) can be exploited. However, these Higgs bosons could be the SM-like Higgs $H_{S M}$ with its known mass of $125 \mathrm{GeV}$, or a lighter NMSSM-specific Higgs boson $H_{S}$ (or a mixture of both, depending on the corresponding branching fractions of the NLSP).

Hence one can look for the decay products of two Higgs bosons in each event, which are dominantly $b \bar{b}$ and subdominantly $\tau^{+} \tau^{-}$for both $H_{S M}$ and $H_{S}$. In [3, 4] we proposed to look for one $b \bar{b}$ pair and one $\tau_{h}^{+} \tau_{h}^{-}$pair together with four hard jets in each event. The requirement of a hadronic $\tau_{h}^{+} \tau_{h}^{-}$pair reduces strongly the background which is dominated by top quark pair production together with one hard jet at the parton level (and possibly fake $\tau_{h}$ 's), and bottom quark pair production with two hard jets at the parton level (and two fake $\tau_{h}$ 's). We assumed, however, that $\tau_{h}^{+} \tau_{h}^{-}$pairs with $\Delta R_{\tau_{h}^{+} \tau_{h}^{-}}$below 0.5 can be identified, although for $\Delta R_{\tau_{h}^{+} \tau_{h}^{-}}$below 0.5 the $2 \tau_{h}$ fake rate becomes larger than the square of the $2 \tau_{h}$ fake rate (which has been taken into account).

The mass(es) of the Higgs boson(s) can be reconstructed from the $b \bar{b}$ pair; two slightly different procedures have been proposed in [3], [4], respectively. In both cases the quantity of interest is the mass $M_{\hat{J}}$ of a "fat" jet $\hat{J}$ constructed out of two (possibly "slim") $b$-tagged jets.

Plots of $M_{\hat{J}}$ are shown in Fig. 4 for two different scenarios (and cuts): In the left panel the binolike NLSP with a mass of $\sim 134 \mathrm{GeV}$ decays to $100 \%$ into the singlino-like LSP with a mass of $\sim 5 \mathrm{GeV}$ and $H_{S M}$, squarks and the gluino have masses of $\sim 1 \mathrm{TeV}$ (still compatible with searches at the run I of the LHC). Cuts on the $p_{T}$ of four jets are chosen as $p_{T}>400,300,200,100 \mathrm{GeV}$. In the right panel the bino-like NLSP with a mass of $\sim 89 \mathrm{GeV}$ decays to $100 \%$ into the singlino-like 
LSP and $H_{S}$ with a mass of $\sim 83 \mathrm{GeV}$. The cuts on the $p_{T}$ of four jets are chosen somewhat softer as $p_{T}>200,100,80,80 \mathrm{GeV}$. The peaks of $M_{\hat{J}}$ are well visible above the background in both cases. (However, for heavier squarks/gluinos the height of the peaks decreases with the squark/gluino production cross sections.
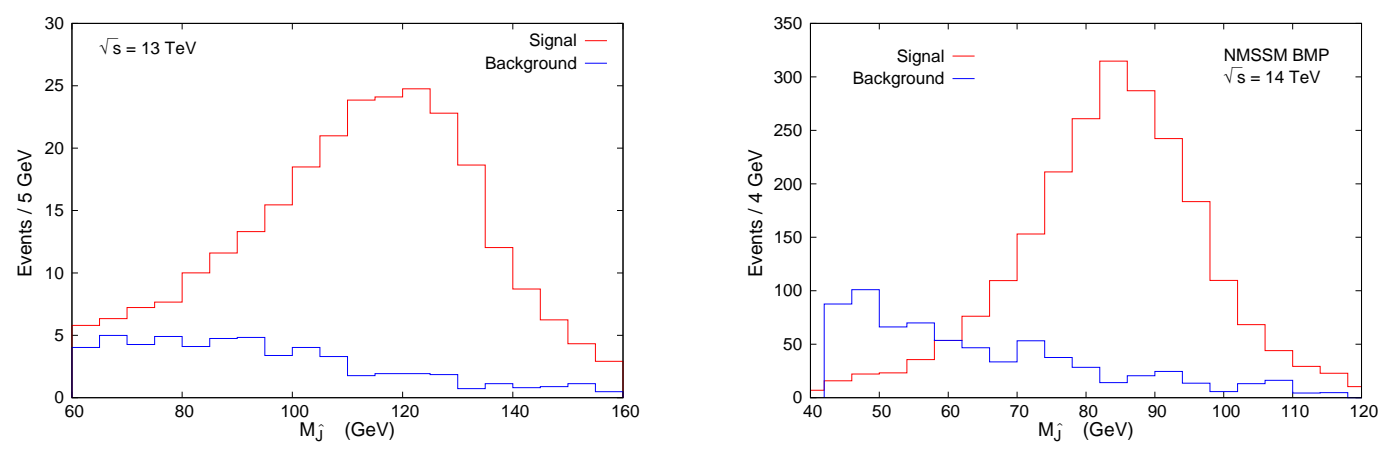

Figure 4: Spectra of $M_{\hat{J}}$ (red) and the background (blue) for the decay NLSP $\rightarrow$ LSP $+H_{S M}$ (left panel) and $\mathrm{NLSP} \rightarrow \mathrm{LSP}+H_{S}, M_{H_{S}} \sim 83 \mathrm{GeV}$ (right panel).

The spectrum of $M_{\hat{J}}$ for a case where the bino-like NLSP with a mass of $\sim 134 \mathrm{GeV}$ decays to $50 \%$ into $H_{S}$ and to 50\% into $H_{S M}$ is shown in Fig. 1 (with cuts as for the left panel in Fig. 4). Now peaks corresponding to both Higgs bosons are visible.

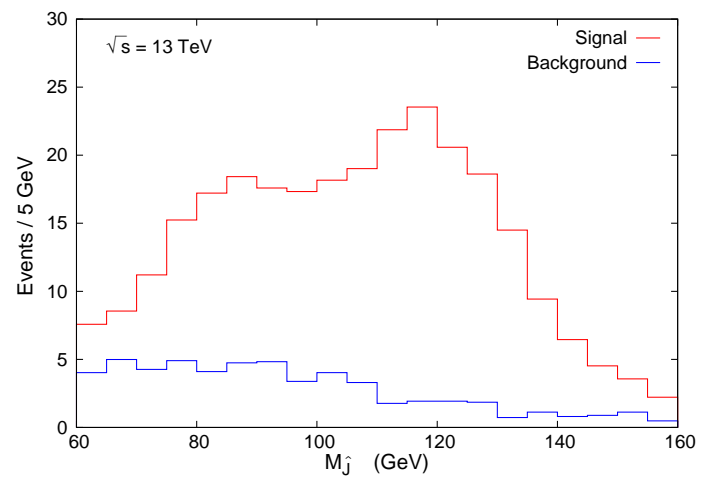

Figure 5: Spectrum of $M_{\hat{J}}$ (red) and the background (blue) for decays of the NLSP to $50 \%$ into $H_{S}$ and to $50 \%$ into $H_{S M}$.

To conclude, in the presence of a light singlino in the NMSSM the standard $E_{T}^{\text {miss }}$ signature for sparticle production can be strongly suppressed. Such scenarios require to re-interprete the absence of excesses at the LHC run I in terms of lower bounds on sparticle masses. One finds that squarks and gluinos with masses below $1 \mathrm{TeV}$ (for both) remain compatible with these constraints.

For the scenarios considered here, instead of $E_{T}^{\text {miss }}$ a Higgs boson is produced in each sparticle decay cascade; possibly the SM-Higgs boson, or a (lighter) NMSSM-specific Higgs boson. Such scenarios are observable through dedicated searches not relying on $E_{T}^{\text {miss }}$, but sensitive to Higgs 
decay products. Such searches may discover supersymmetry together with an additional Higgs boson!

\section{References}

[1] G. Aad et al. [ATLAS Collaboration], "Search for squarks and gluinos with the ATLAS detector in final states with jets and missing transverse momentum using $\sqrt{s}=8 \mathrm{TeV}$ proton-proton collision data," JHEP 1409 (2014) 176 [arXiv:1405.7875 [hep-ex]].

[2] U. Ellwanger, C. Hugonie and A. M. Teixeira, "The Next-to-Minimal Supersymmetric Standard Model,” Phys. Rept. 496 (2010) 1 [arXiv:0910.1785 [hep-ph]].

[3] U. Ellwanger and A. M. Teixeira, "NMSSM with a singlino LSP: possible challenges for searches for supersymmetry at the LHC," JHEP 1410 (2014) 113 [arXiv:1406.7221 [hep-ph]].

[4] U. Ellwanger and A. M. Teixeira, "Excessive Higgs pair production with little MET from squarks and gluinos in the NMSSM," JHEP 1504 (2015) 172 [arXiv:1412.6394 [hep-ph]]. 